\title{
Controlling Polarization at Insulating Surfaces: Quasiparticle Calculations for Molecules Adsorbed on Insulator Films
}

\author{
Christoph Freysoldt, ${ }^{1}$ Patrick Rinke, ${ }^{1,2}$ and Matthias Scheffler ${ }^{1}$ \\ ${ }^{1}$ Fritz-Haber-Institut der Max-Planck-Gesellschaft, Faradayweg 4-6, 14195 Berlin, Germany \\ ${ }^{2}$ Materials Department, University of California, Santa Barbara, California 93106, USA
}

(Received 3 March 2009; published 31 July 2009)

\begin{abstract}
By means of quasiparticle-energy calculations in the $G_{0} W_{0}$ approach, we show for the prototypical insulator-semiconductor system $\mathrm{NaCl} / \mathrm{Ge}(001)$ that polarization effects at the interfaces noticeably affect the excitation spectrum of molecules adsorbed on the surface of the $\mathrm{NaCl}$ films. The magnitude of the effect can be controlled by varying the thickness of the film, offering new opportunities for tuning electronic excitations in, e.g., molecular electronics or quantum transport. Polarization effects are visible even for the excitation spectrum of the $\mathrm{NaCl}$ films themselves, which has important implications for the interpretation of surface science experiments for the characterization of insulator surfaces.
\end{abstract}

DOI: 10.1103/PhysRevLett.103.056803

PACS numbers: 73.20.- r, 71.20.Ps

On the nanoscale, materials often reveal extraordinary features. To harness this potential it is essential to grow or manufacture nanostructures in a controlled way. Ultrathin insulating films are an example for which this has been achieved on metal and semiconductor surfaces. We have recently shown that these films develop new and unique properties as their thinness approaches the limit of a few atomic layers and that such supported ultrathin films should be regarded as new nanosystems in their own rights [1]. Here we go one step further and demonstrate by means of first principles calculations that control over the film thickness means control over the polarization of the film. This in turn gives access to properties on the film's surface, for example, the energy levels of molecular adsorbates, which are relevant in the context of, e.g., catalysis, molecular electronics, or quantum transport.

The fact that ultrathin insulator films offer a new perspective of control at the nanoscale is increasingly being recognized. Repp et al., for example, have recently demonstrated that gold atoms adsorbed on $\mathrm{NaCl} / \mathrm{Cu}$ can be reversibly switched between the neutral and negative charge state [2]. Alternatively, the charge state of Au atoms on $\mathrm{MgO}$ films can be controlled by the film thickness [3]. For planar molecules adsorbed on $\mathrm{Cu}$-supported $\mathrm{NaCl}$ films, the molecular orbitals can be resolved spatially and energetically [4,5] by scanning tunneling microscopy (STM) and spectroscopy (STS), and even reactions can be followed [6]. Interestingly, STS experiments performed on pentacene molecules adsorbed on $\mathrm{NaCl} / \mathrm{Cu}(111)$ show a significant influence of the film thickness on the molecular gap [5].

Ultrathin insulator films have also developed into highly valuable and intensively studied model systems for characterizing insulating surfaces. The study of insulator surfaces has proven difficult due to their lack of conductivity, which severely limits the range of applicable surface science techniques. Ultrathin insulator films grown on con- ducting substrates offer a solution to this dilemma because the films can exchange electrons with the substrate by tunneling [7,8]. Caution has to be applied, however, when transferring thin-film results to the surfaces of technological interest. The properties of ultrathin films may deviate considerably from those of macroscopic films [1], and the excitation spectrum may be affected by the polarization effects presented here.

In this Letter we address both of these points by means of $G_{0} W_{0}$ quasiparticle-energy calculations $[9,10]$ for the example of a prototypical insulator-semiconductor interface $[\mathrm{NaCl} / \mathrm{Ge}(001)]$ and $\mathrm{CO}$ as a model adsorbate. Supported $\mathrm{NaCl}$ films are well-behaved model systems for studying the properties of insulator surfaces [6,1113]. Although they are mostly grown on metals, notably $\mathrm{Cu}[2,13], \mathrm{Ge}(001)$ is the substrate of choice for studying insulator-semiconductor interfaces $[11,12,14-16]$. In recent years, these films have also attracted increasing interest in the context of STM and STS studies of atomic and molecular adsorbates [2-6].

We use density-functional theory (DFT) in the localdensity approximation (LDA) to determine the atomic structure of $\mathrm{NaCl}$ films on $\mathrm{Ge}(001)$. The electronic excitation spectrum is calculated with many-body perturbation theory in the $G W$ approach as perturbation to the LDA ground state (henceforth denoted $G_{0} W_{0} @$ LDA). The $G W$ approach not only has become the method of choice for calculating quasiparticle excitations in solids $[9,10]$ as probed in STS or direct and inverse photoemission, but also includes long-range polarization effects. At a surface or an interface, the abrupt change in dielectric constant gives rise to a net buildup of charge, so-called image charges. This net polarization acts back on the additional charge and increases in strength with decreasing distance between the additional charge and the interface. These polarization effects are absent from the most common density functionals (such as the local-density or general- 
ized gradient approximation, exact-exchange, and hybrid functionals), but enter the $G W$ self-energy $(\Sigma=i G W)$ through the screened Coulomb potential $W$. The application of the $G W$ method is therefore necessary to capture these effects [17-20].

Polarization or image effects are present at any surface or interface, but are most commonly associated with metal surfaces, where the ratio in dielectric constants is largest. In supported ultrathin films, however, a charged excitation [e.g., an electron added to the $2 \pi^{*}$ level of $\mathrm{CO}$ on $\mathrm{NaCl} / \mathrm{Ge}(001)]$ polarizes two interfaces [vacuum/ $\mathrm{NaCl}$ and $\mathrm{NaCl} / \mathrm{Ge}(001)]$. The combination of dielectric constants and film thickness therefore controls the strength of the polarization effects from the semiconductor-insulator interface to the insulator surface.

Before we address the polarization effects at the $\mathrm{NaCl} / \mathrm{Ge}$ interface in detail, we briefly describe its atomic structure, which had not been determined previously. The DFT-LDA calculations were performed with the SFHINGX code [21]. We employ a plane-wave basis set (40 Ry cutoff) and norm-conserving pseudopotentials. The $\mathrm{NaCl} / \mathrm{Ge}$ system is modeled in the repeated-slab approach with a 6layer Ge slab at the experimental lattice constant (saturated by hydrogen atoms on the bottom side) plus a varying number $\mathrm{NaCl}$ layers on the top side [22]. Increasing the Ge thickness to 12 layers produces no significant change in the atomic or electronic structure of the $\mathrm{NaCl}$ films. In agreement with experimental indications [16], we find that the Ge dimers of the clean $\mathrm{Ge}(001)$ surface prevail below the $\mathrm{NaCl}$ film, giving rise to a $2 \times 1$ surface lattice [11]. The $\mathrm{Ge}$ dimers below the $\mathrm{NaCl}$ film remain asymmetric, but with a smaller tilting angle $\left(10^{\circ}\right)$ compared to the free surface $\left(19^{\circ}\right)$. The adhesion of the film is dominated by the electrostatic interaction between the ions in the film and the partial charges developing at the buckled-dimer surface of $\mathrm{Ge}(001)$. The relaxation pattern in the bottom layer follows the electrostatic profile of the Ge surface, which attracts the ions next to the dimer, but repels those above the interdimer troughs (cf. Fig. 1). The corrugation in the higher layers is induced by the bottom layer and quickly flattens out as the thickness increases.

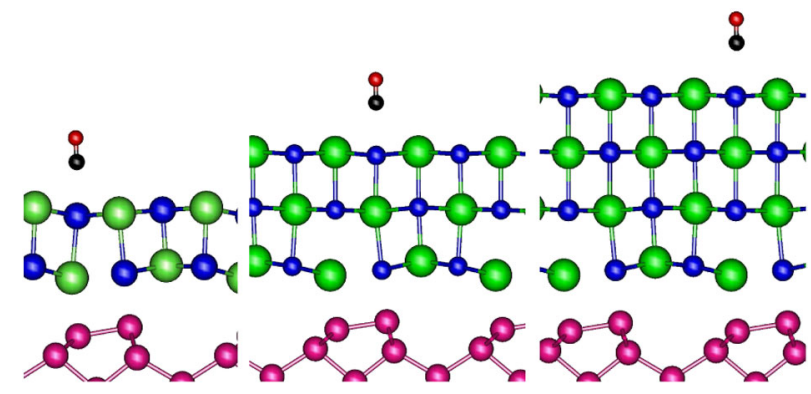

FIG. 1 (color online). Structure of $\mathrm{NaCl}$ films (2-4 layers) on $\mathrm{Ge}(001)$ with a $\mathrm{CO}$ adsorbate. The spheres denote the atoms: Ge [medium-sized light gray (magenta)], $\mathrm{Na}$ [medium dark gray (blue)], $\mathrm{Cl}$ [large light gray (green)], $\mathrm{C}$ (small black), $\mathrm{O}$ [small gray (red)].
Next, we use the adsorption of a small molecule to probe the effect of the interface polarization at the film's surface. For this purpose, we placed a single $\mathrm{CO}$ molecule in the $2 \times 1$ surface unit cell (we have no indications for relevant changes at lower coverages). $\mathrm{CO}$ physisorbs perpendicular to the $\mathrm{NaCl}(001)$ surface with the $\mathrm{C}$ end down (cf. Fig. 1). The $\mathrm{CO}$ axis tilts along (110), i.e., the Ge dimer, by $2.6^{\circ}$ for 2 monolayer (ML) $\mathrm{NaCl}, 0.7^{\circ}$ (3 ML), and $0.1^{\circ}$ (4 ML), respectively. The adsorption energy of $0.28 \mathrm{eV}$ is the same for the two inequivalent $\mathrm{Na}$ sites at the surface and does not depend on the film's thickness to within $0.01 \mathrm{eV}$. It also agrees with the value for a thick freestanding $\mathrm{NaCl}$ slab. The molecular states give rise to flat bands in the band structure and do not hybridize with $\mathrm{NaCl}$ states. In the following, we focus on the molecular gap (given by the $5 \sigma-2 \pi^{*}$ splitting) at the $\bar{\Gamma}$ point.

The $G_{0} W_{0}$ calculations for the electron addition and removal spectra were performed with the GWST code [23-25]. For the correlation (exchange) self-energy, a 14 Ry (28 Ry) plane-wave cutoff and a $6 \times 3 \times 1$ $\mathbf{k}$-point sampling was used. State summations included 2500 bands ( $81 \mathrm{eV}$ above the Fermi level). To correct for artificial polarization effects in the repeated-slab approach, a "finite vacuum" correction was applied [26].

In Fig. 2, we compare the molecular gap of $\mathrm{CO}$ computed for LDA and $G_{0} W_{0} @ \mathrm{LDA}$ for films of 2-4 ML thickness. At the level of LDA, we observe a small reduction of the molecular gap with increasing film thickness due to the thickness-dependent structural changes in the surface of the $\mathrm{NaCl}$ film. The $G_{0} W_{0}$ corrections reverse this trend and introduce a significant increase in the quasiparticle gap from $12.48 \mathrm{eV}$ (for $2 \mathrm{ML}$ ) to $12.80 \mathrm{eV}$ (4 ML). The other $\mathrm{CO}$ orbitals exhibit analogous thicknessdependent shifts (not shown). These gaps are significantly smaller than for $\mathrm{CO}$ on a pure $\mathrm{NaCl}$ surface $(13.1 \mathrm{eV}$ for $6 \mathrm{ML} \mathrm{NaCl}$, no Ge) or the free molecule $(15.1 \mathrm{eV})$. Similar reductions have been found in $G_{0} W_{0}$ calculations for molecules adsorbed on insulators [27], metals [28], or semimetals [29] and are a result of surface polarization or charge transfer to the molecule [30].

We now demonstrate that the reduction of the $5 \sigma-2 \pi^{*}$ splitting is caused by polarization effects at the two inter-
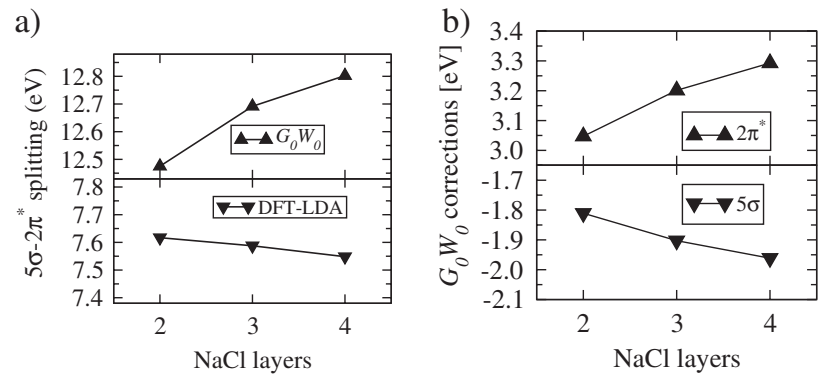

FIG. 2. (a) $5 \sigma-2 \pi^{*}$ splitting of $\mathrm{CO}$ on $\mathrm{NaCl} / \mathrm{Ge}(001)$ at the LDA and $G_{0} W_{0} @$ LDA levels of theory, and (b) $G_{0} W_{0}$ corrections to the $5 \sigma$ and $2 \pi^{*}$ molecular orbitals as a function of the $\mathrm{NaCl}$ film thickness. 
faces. Inspection of Fig. 2(b) reveals that the thicknessdependent changes are of similar magnitude, but opposite sign. Such a behavior is characteristic for long-range polarization effects $[26,29]$. To illustrate this, we split the electron addition or removal into two steps. First, the charged state is created on a free molecule. Second, we consider the electronic polarization of the $\mathrm{NaCl} / \mathrm{Ge}$ substrate. It lowers the energy of the charged state $E_{N \pm 1}$ by $\frac{1}{2} q \Delta V$, where $\Delta V$ is the polarization-induced change in the electrostatic potential and $q$ the charge. The factor $\frac{1}{2}$ arises from the adiabatic switching on of the polarization [26]. The energy of the hole state $\left(E_{N}-E_{N-1}\right)$ thereby increases whereas that of the electron state $\left(E_{N+1}-E_{N}\right)$ becomes smaller.

To estimate $\Delta V$ outside the supported $\mathrm{NaCl}$ films, the $\mathrm{Ge}$ substrate, the $\mathrm{NaCl}$ film, and the vacuum region are replaced by homogeneous dielectric media $(\varepsilon=14,2.8$, and 1 , respectively) with abrupt interfaces. The thickness of the $\mathrm{NaCl}$ region is taken to be $2.8 \AA$ /layer. The effective polarization is then computed using the image-charge method. For $\Delta V$, we then take the value of the image potential at the position of the $\mathrm{CO}$ molecule outside the surface. Since this position is somewhat ambiguous due to the spatial extent of the $\mathrm{CO}$ orbitals, we determine it by requiring that the reduction of the $5 \sigma-2 \pi^{*}$ splitting of $2 \mathrm{eV}$ at the bare $\mathrm{NaCl}$ surface is reproduced by the model. This yields a value of $1.5 \AA$, and the model then gives $5 \sigma-2 \pi^{*}$ splittings of $12.6,12.7$, and $12.8 \mathrm{eV}$ for the 2,3 , and $4 \mathrm{ML}$ films, respectively, in good agreement with the values from our $G_{0} W_{0} @$ LDA calculations.

On the experimental side, a gap reduction as a function of film thickness has been reported for STS experiments on pentacene molecules adsorbed on $\mathrm{NaCl} / \mathrm{Cu}(111)$ [5]. In STS, the molecular states give rise to tunneling resonances, and the observed tunneling gap amounts to 3.3, 4.1, and $4.4 \mathrm{eV}$ for $\mathrm{NaCl}$ films of 1,2 , and $3 \mathrm{ML}$ in thickness (the gap of pentacene in the gas phase is $5.3 \mathrm{eV}$ ). The overall trend as well as the magnitude agree very well with our $G_{0} W_{0} @ \mathrm{LDA}$ calculations for $\mathrm{CO} / \mathrm{NaCl} / \mathrm{Ge}$ considering that the ratio of dielectric constants is much larger in the $\mathrm{NaCl} / \mathrm{Cu}$ case. A tunneling resonance gap will be hard to observe experimentally for $\mathrm{CO} / \mathrm{NaCl} / \mathrm{Ge}$, however, because the highest occupied molecular orbital $(5 \sigma)$ is located $\sim 8.5 \mathrm{eV}$ below the Ge valence band maximum in $G_{0} W_{0} @ \mathrm{LDA}$ and thus even below the $\mathrm{NaCl}$ valence band (4-7 eV below the Ge valence band maximum, cf. Fig. 3).

Our results highlight that surface and interface polarization effects are important for adsorbed atoms and molecules. Supported ultrathin films thereby offer unprecedented opportunities for controlling these effects by tailoring both the film's thickness and dielectric constant to the desired properties. These additional parameters may expedite the design of devices in molecular electronics or quantum transport, where the distance of a molecular state to the Fermi level is an important quantity.

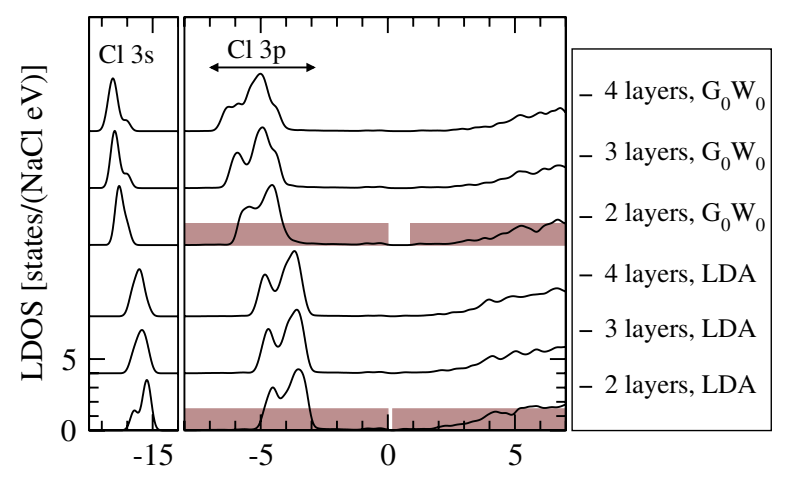

FIG. 3 (color online). DOS inside the supported $\mathrm{NaCl}$ films of 2-4 ML thickness at the LDA and the $G_{0} W_{0} @$ LDA level. The shaded region indicates the energy position of the Ge bands. The valence band maximum of the Ge slab defines the energy zero. (LDOS is the local density of states.)

We now demonstrate that the same polarization effects also affect excitations inside the ultrathin film and discuss the implications of this for the characterization of insulator surfaces. Figure 3 shows the density of states (DOS) projected onto the $\mathrm{NaCl}$ film for films with 2-4 ML for LDA with and without the $G_{0} W_{0}$ corrections. The most important change when comparing the LDA and $G_{0} W_{0}$ DOS is the shift of the $\mathrm{NaCl}$ bands [31] relative to the $\mathrm{Ge}$ states. This is not too surprising, as the $G_{0} W_{0}$ corrections to the bulk band gaps are much larger for $\mathrm{NaCl}(3.3 \mathrm{eV})$ than for Ge $(0.7 \mathrm{eV})$. Including the $G_{0} W_{0}$ corrections, the top of the film's valence band lies $\sim 4.2 \mathrm{eV}$ below that of the Ge substrate in excellent agreement with ultraviolet photoelectron spectroscopy [32]. More remarkable, however, is the change in the shape of the $\mathrm{NaCl}$-derived features when going from LDA to $G_{0} W_{0} @ \mathrm{LDA}$ and from 2 to 4 layers. This indicates that the $G_{0} W_{0}$ shifts for the $\mathrm{NaCl}$ states are not uniform and that corrections derived from bulk calculations are not easily transferable to thin films. Excited states are instead subject to additional thickness-dependent and substrate-specific changes that are neither encompassed by a ground-state perspective nor easily derivable from bulk $G_{0} W_{0}$ calculations for the separate fragments alone. These thickness-dependent variations should be observable in high-resolution spectroscopic experiments.

In LDA the DOS of bulk $\mathrm{NaCl}$ (not shown) is already attained at a thickness of only three layers. A similar behavior has previously been observed for ultrathin silica, hafnia, and alumina films [1]. However, this is no longer the case when charged excitations (e.g., photoemission or tunneling) are treated appropriately. The $G_{0} W_{0} @$ LDA calculations demonstrate clearly that the DOS of ultrathin films differs from that of bulk $\mathrm{NaCl}$ (which is identical in shape to the LDA DOS for a 4-layer film). This implies that caution has to be applied when interpreting spectroscopic results. Spectra of ultrathin films are not representative of bulk samples.

The nonuniform $G_{0} W_{0}$ shifts are also a result of the interface polarization effects. The position dependence of 


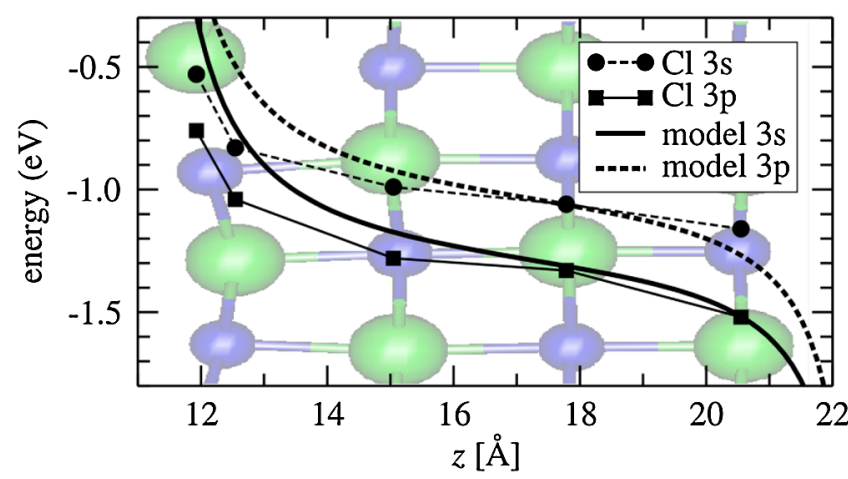

FIG. 4 (color online). Comparison of the extrapolated $G_{0} W_{0}$ orbital shifts for the 4-layer $\mathrm{NaCl}$ film to the image potential of the dielectric slab model (cf. text).

the self-energy $(\Sigma=i G W)$ can be understood with the same dielectric slab model introduced above. Solving the image-charge model yields the induced potential $\Delta V(z)$ as a function of the position in the film $z$. From this, we build the following model self-energy for the occupied $\mathrm{NaCl}$ states:

$$
\Sigma(z)=\Delta \Sigma-\frac{1}{2} \Delta V(z)
$$

The constant $\Delta \Sigma$ encompasses all $G W$ self-energy effects that are not due to image effects. Its actual value is not important for analyzing the position dependence of the self-energy. Based on our $G_{0} W_{0}$ calculations, we estimate it to be $-1.05 \mathrm{eV}$ for $\mathrm{Cl} 3 s$ and $-1.3 \mathrm{eV}$ for $\mathrm{Cl} 3 p$. In order to assess the local variation of the self-energy in the full $G_{0} W_{0}$ calculation, the valence bands are projected onto atomic orbitals. For states that are predominantly composed of orbitals from a single atomic layer $(>80 \%)$ we observe a linear dependence between the $G_{0} W_{0}$ correction and the projection weight. By extrapolating to $100 \%$, the orbital-dependent quasiparticle corrections shown in Fig. 4 are obtained. The variation of the quasiparticle shifts throughout the $\mathrm{NaCl}$ film $\left(G_{0} W_{0}\right.$ DOS in Fig. 3) is reproduced well by a projection-weighted sum of these localorbital contributions. In Fig. 4, we compare the extrapolated shifts to our model self-energy. The good agreement illustrates that the interface polarization effects in the $\mathrm{Ge} / \mathrm{NaCl}$ system are indeed the cause for the position dependence in the $G_{0} W_{0}$ self-energy.

We acknowledge fruitful discussions with Philipp Eggert and the Nanoquanta Network of Excellence (NMP4-CT-2004-500198) for financial support.

[1] C. Freysoldt, P. Rinke, and M. Scheffler, Phys. Rev. Lett. 99, 086101 (2007).

[2] J. Repp, G. Meyer, F. E. Olsson, and M. Persson, Science 305, 493 (2004).
[3] M. Sterrer, T. Risse, U.M. Pozzoni, L. Giordano, M. Heyde, H.-P. Rust, G. Pacchioni, and H.-J. Freund, Phys. Rev. Lett. 98, 096107 (2007).

[4] J. Repp, G. Meyer, S. M. Stojković, A. Gourdon, and C. Joachim, Phys. Rev. Lett. 94, 026803 (2005).

[5] J. Repp and G. Meyer, Appl. Phys. A 85, 399 (2006).

[6] J. Repp, G. Meyer, S. Paavilainen, F. E. Olsson, and M. Persson, Science 312, 1196 (2006).

[7] H.-J. Freund, Surf. Sci. 500, 271 (2002).

[8] D. Goodman, J. Catal. 216, 213 (2003).

[9] W. G. Aulbur, L. Jönsson, and J. W. Wilkins, in Solid State Physics, edited by H. Ehrenreich and F. Spaepen (Academic, New York, 2000), Vol. 54, p. 1.

[10] P. Rinke, A. Qteish, J. Neugebauer, C. Freysoldt, and M. Scheffler, New J. Phys. 7, 126 (2005).

[11] S. Fölsch, U. Barjenbruch, and M. Henzler, Thin Solid Films 172, 123 (1989).

[12] V. Zielasek, T. Hildebrandt, and M. Henzler, Phys. Rev. B 62, 2912 (2000).

[13] F.E. Olsson, M. Persson, J. Repp, and G. Meyer, Phys. Rev. B 71, 075419 (2005).

[14] C. Schwennicke, J. Schimmelpfennig, and H. Pfnür, Surf. Sci. 293, 57 (1993).

[15] K. Glöckler, M. Sokolowski, A. Soukopp, and E. Umbach, Phys. Rev. B 54, 7705 (1996).

[16] V. Zielasek, T. Hildebrandt, and M. Henzler, Phys. Rev. B 69, 205313 (2004).

[17] I. D. White, R. W. Godby, M. M. Rieger, and R. J. Needs, Phys. Rev. Lett. 80, 4265 (1998).

[18] G. Fratesi, G. P. Brivio, P. Rinke, and R. W. Godby, Phys. Rev. B 68, 195404 (2003).

[19] M. Rohlfing, N.-P. Wang, P. Krüger, and J. Pollmann, Phys. Rev. Lett. 91, 256802 (2003).

[20] P. Rinke, K. Delaney, P. García-González, and R. W. Godby, Phys. Rev. A 70, 063201 (2004).

[21] http://www.sphinxlib.de.

[22] The $2 \times 1$ surface cell has 2 atoms per Ge layer ( 4 per $\mathrm{NaCl}$ layer). A vacuum region of $\sim 10 \AA$ proved sufficient to decouple the slabs. While bulk Ge is a metal in LDA, the 6-layer slab has a gap of $0.2 \mathrm{eV}$ due to quantum confinement, which simplifies the $G_{0} W_{0}$ calculations.

[23] M. Rieger et al., Comput. Phys. Commun. 117, 211 (1999).

[24] L. Steinbeck et al., Comput. Phys. Commun. 125, 105 (2000).

[25] C. Freysoldt et al., Comput. Phys. Commun. 176, 1 (2007).

[26] C. Freysoldt, P. Eggert, P. Rinke, A. Schindlmayr, and M. Scheffler, Phys. Rev. B 77, 235428 (2008).

[27] M. Rohlfing, Int. J. Quantum Chem. 80, 807 (2000).

[28] X. Ren, P. Rinke, and M. Scheffler, Phys. Rev. B 80, 045402 (2009).

[29] J. B. Neaton, M. S. Hybertsen, and S. G. Louie, Phys. Rev. Lett. 97, 216405 (2006).

[30] K. S. Thygesen and A. Rubio, Phys. Rev. Lett. 102, 046802 (2009).

[31] Since a meaningful analysis of the NaCl's conduction bands is aggravated by their very strong hybridization with Ge states, we focus on the valence states only.

[32] U. Barjenbruch, S. Fölsch, and M. Henzler, Surf. Sci. 211212, 749 (1989). 